\title{
Review on Angiogenesis and Its Importance in Tumor Progression
}

\section{DrDandena Vinay Kumar MDS, Dr. Shaktidar PR MDS, DrHemavathy S MDS, DrSahana NS MDS}

\begin{abstract}
Angiogenesis is the process of formation of new capillary blood vessels whichis one of the most pervasive and fundamentally essential biological processes encountered in mammalian organisms. Angiogenesis is an important event in a variety of physiological settings, such as embryonic development, chronic inflammation,wound repair and in pathological states like tumor progression. Here we are describing about the main biological events involved in angiogenesis and their importance in cancer progression
\end{abstract}

Key words: Angiogenesis, Cancer, Blood Vessels, Wound healing.

\section{Biology Of The Healthy Vasculature}

The most essential component of blood vessels is the endothelial cell (EC). Every vessel, from the aorta down to the smallest capillaries, consists of a monolayer of EC (called the endothelium), arranged in a mosaic pattern around a central lumen, through which blood canflow. In the smallest vessels, a cross-section of the endothelium may consist of a single EC, which has wrapped around to form a lumen. The endothelium controls the passage of nutrients, white blood cells and other materials between the bloodstreamand the tissues. The healthy endothelium represents a highly stable population ofcells:cell-cell connections are tight and the cell turnover periodis measured in months or years. Outside the endothelium is an extracellular lining called the basement membrane, separating the EC from the surrounding connective tissue. This is composed of protein fibres, mainly laminin and collagen, and may also contain peri-endothelial support cells. These are pericytes in the microvasculature (capillaries) and smooth muscle cells in larger vessels. The basement membrane serves as a scaffold on which the EC rest and helps to maintain the endothelium in its quiescent state.Cell-cell contacts and cell-basement membrane contacts, mediated by adhesion molecules (such as cadherins and integrins, respectively), are extremely important and loss of either or both can lead to local destabilisation of the endothelium and EC apoptosis. The peri-endothelial cells play a particularly important role in maintaining blood vessels in the stable state, and may be involved in the regulation of blood flow. ${ }^{1}$

Larger vessels have a thick wall of smooth muscle outside the basement membrane, whereas capillaries consist only of the endothelium, basement membrane and pericytes. We are primarily concerned with capillaries (the microvasculature), as opposed to larger vessels, since it is the former that are involved in angiogenesis; the latter can form only via remodelling of the microvasculature following endothelial branching and tube formation. ${ }^{1}$

The formation of blood vessels can be divided into two separate processes. Vasculogenesis is the in situ differentiation of endothelial cells from haemangioblasts (precursors of EC) and their subsequent organisation into a primitive vascular network. Angiogenesis is the sprouting, splitting and remodelling of existing vessels. Vasculogenesis is confined to early embryonic development and is responsible for the formation of the primary vasculature, including the main vessels of the heart and lungs. Angiogenesis subsequently extends the circulation into previously avascular regions by the controlled migration and proliferation of EC. ${ }^{1,2,3,4}$.

\section{Physiological Control Of Angiogenesis}

The entire skin surface overlies a vast network of capillary blood vessels. Beneath the epidermis, each cell exists no greater than $200 \mu \mathrm{m}$ from the nearest capillary, the diffusion distance of oxygen. Most blood vessels are formed during fetal development, but adult tissues can induce angiogenesis in response to injury. This capability is governed by pro- and antiangiogenic factors present throughout the body.

Pro-angiogenic factors consist of a diverse group of molecules including thrombin, fibrinogen fragments, thymosin beta 4, and growth factors. Angiogenic growth factors are proteins that circulate in the bloodstream, are stored in platelets and inflammatory cells, and are sequestered within the extracellular matrix. The production of many of these factors is regulated by genes expressed in response to hypoxia and inflammation, such as hypoxia-inducible factors (HIF) and cyclooxygenase-2 (COX-2), ${ }^{2,5,6}$

Angiogenesis inhibitory factors suppress blood vessel growth. Some inhibitors circulate in the bloodstream at low physiological levels, while others are stored in the extracellular matrix surrounding blood vessels. A precise physiological balance exists between angiogenesis stimulators and endogenous inhibitors, 
such that vascular growth is normally suppressed. Immediately following injury, however,angiogenic stimuli are released into the wound bed, and a shift occurs in the balance of regulators favoring vascular growth. ${ }^{7}$

\section{Tumour-Induced Angiogenesis}

Angiogenesis is absolutely essential for embryonic growth, tissue growth and repair. Nevertheless, its occurrence is highly restricted and, in the healthy adult, angiogenesis is confined to the female reproductive cycle (Reynolds et al., 1992). Angiogenesis can, however, be induced under certain pathological conditions, such as rheumatoid arthritis (Walsh, 1999), wound healing (Hunt et al., 1984), diabetic retinopathy (Sharp, 1995) and solid tumour growth (Folkman, 1971). ${ }^{1,8}$

A hallmark of pathologic angiogenesis is persistent growth of blood vessels (ie, sustained neovascularization). Angiogenesis that continues for months or years supports the progression of many neoplastic and nonneoplastic diseases. However, both physiologic and pathologic angiogenesis are usually focal. An angiogenic focus appears as only a tiny fraction or a small "hot spot" of proliferating and migrating endothelial cells that arise from a monolayer of resting endothelium.

\section{Historical overview:}

For more than 100 years, tumors had been observed to be more vascular than normal tissues. This tumor hyperemia observed during surgery was explained by simple dilation of existing host blood vessels. Vasodilation was generally thought to be a side effect of metabolites or of necrotic tumor products escaping from the tumor.

Reports suggested that tumor hyperemia could be related to new blood vessel growth; that is, to neovascularization and not solely to vasodilation. A 1939 paper showed that there was neovascularization of a wound in a transparent chamber in a rabbit ear regressed completely after the wound healed,a tumor implant in the chamber was associated with accelerated growth of new capillary blood vessels. The other two reports, in 1945 and 1947, demonstrated that new vessels in the neighborhood of a tumor implant arose from host vessels and not from the tumor itself. These papers notwithstanding, debate continued in the literature for two more decades about whether a tumor could expand to a large size (centimeters) by simply living on preexisting vessels. Even among the few investigators who accepted the concept of tumor-induced neovascularization, it was generally assumed that this vascular response was an inflammatory reaction, a side effect of tumor growth, not a requirement for tumor growth.

In 1971 it was by folkman suggested that the growth of a tumour beyond a diameter of approximately $2 \mathrm{~mm}$ is dependent on its ability to recruit new blood vessels, it was not known how this process might take place, nor how the tumour might induce it. It was postulated that the tumour secretes some diffusible substance, named tumour angiogenesis factor (TAF), which would stimulate the growth of new capillaries. ${ }^{1,9,11 .}$

Tumour cells typically form a contiguous growing cluster, which is reliant on passive diffusion for the supply of oxygen and nutrients and the removal ofwaste products. The tumours need for nutrients grows in proportion to its volume, but its ability to absorb diffusing substances from the surrounding tissue is proportional to its surface area. This imposes a maximum size to which the tumour can grow before it experiences nutrient deficiency.Some of the tumour cells (usually those towards the centre of the tumour, where nutrient levels are at their lowest) will not have sufficient nutrients to continue to proliferate and will become quiescent. If the nutrient supply is not improved, necrosis (cell death caused by insufficient nutrition or injury) will set in, leading to the developmentof necrotic core of dead cells (Sutherland, 1986). ${ }^{1,8}$.

\section{The tumour thus develops a three-layer structure:}

1) A necrotic core,

2) Surrounded by a layer of quiescent cells,

3) Which is in turn surrounded by a thin proliferating rim(Folkman andHochberg,1973).

\section{Emergence from the Dormant Phase and the Angiogenic Switch:}

The existence of the quiescent layer presents a problem for treatments, such as chemotherapy, that are based on the intravenous administration of an agent that is toxic toproliferating cells. The proliferating rim may be eradicated, but the cells underneath will not be affected and will emerge from quiescence to become proliferating cells. ${ }^{1}$

Hypoxic tumour cells are known to produce growth factors, including VEGF they may also produce certain endogenous inhibitors of angiogenesis, such as Transforming growth factor-beta (TGF- $\beta$ ).Moreover, macrophages (cells of the immune system), which congregate in the region of the abnormal growth, respond to the presence of the tumour and its secretions by producing both pro and anti-angiogenic substances. These molecules diffuse through the tissue and will be detected by the EC of proximal capillaries. 
There are a large number of pro-angiogenic and antiangiogenic factors, some of which are produced by the tumour, some of which are produced by host cells in response to the tumour, and some of which are present in normal tissue. It is a shifting of the balance from the anti- to the pro-angiogenic factors (the so-called 'angiogenic switch') that causes the transition from the dormant to the angiogenic phase. This switch is a highly complex process, which is not yet fully understood, but hypoxia (oxygen deficiency) in the tumour is thought to be an important factor, stimulating production of pro-angiogenic molecules by the tumour cells (Shweiki et al., 1992). ${ }^{1,2}$.

\section{Initiation, Proliferation And Capillary Tube Formation:}

On receiving a net angiogenic stimulus, EC in capillaries near the tumour become activated: they loosen the normally tight contacts with adjacent cells and secrete proteolytic enzymes (or proteases), whose collective effect is to degrade extracellular tissue. There are a large number of such enzymes, which may be broadly divided into matrix metalloproteases (MMPs) and the plasminogen activator (PA)/plasmin system. The MMPs are capable of digesting different protein types and may be subdivided accordingly into collagenases, gelatinases, stromelysins, matrelysins and membrane-type MMPs. PAs activate the widely expressed, but inactive substance, plasminogen, into the broad-spectrum protease, plasmin. Both of these families of proteases have an associated class of inhibitors. MMPs are inhibited by tissue inhibitors of metalloproteases (TIMPs). PAs are inhibited by plasminogen activator inhibitor (PAI), which is also expressed by fibroblasts and activated EC (Pepper et al., 1992)

The first target of the proteases produced by the EC is the basement membrane. When this has been sufficiently degraded, the EC are able to move through the gap in the basement membrane and into the ECM. Neighbouring EC move in to fill the gap and may subsequently follow the leading cells into the ECM .

The first function of the angiogenic growth factors, therefore, is to stimulate the production of proteases by EC. This is a key step in the angiogenic cascade because, in the absence of proteolytic activity, the EC are hemmed in by the basement membrane and will be unable to escape from the existing capillary (parent vessel).

Following extravasation of endothelial cells they proliferate and initially take the form of solid strands of cells, but the EC subsequently form a central lumen, thereby creating the necessary structure for a new blood vessel.

\section{Experimental evidence}

By the mid-1980s, considerable experimental evidence had been assembled to support the hypothesis that tumor growth is angiogenesis dependent. The idea could now be stated in its simplest terms: "Once tumor take has occurred, every further increase in tumor cell population must be preceded by an increase in new capillaries that converge upon the tumor" The hypothesis predicted that if angiogenesis could be completely inhibited, tumors wouldbecome dormant at a small, possibly microscopic, size. It forecast that whereas the presence of neovascularization would be necessary, but not sufficient for expansion of a tumor, the absence of neovascularization would prevent expansion of a primary tumor mass beyond

1 to $2 \mathrm{~mm}^{3}$ and restrict a metastasis to a microscopic dormantlesion. Most nonneovascularized tumors are not clinically detectable, with the exception of surface lesions on the skin or the external mucous membranes. The hypothesis that tumors are angiogenesis dependent is supported by biologic and pharmacologic evidence and proved by genetic evidence. ${ }^{10}$

\section{Biologicevidence:}

Tumors implanted into subcutaneous transparent chambers grow slowly before vascularization, and tumor volume increases linearly. After vascularization, tumor growth is rapid and tumor volume may increase exponentially.

Tumor growth in the avascular rabbit cornea proceeds slowly at a linear rate, but converts to exponential growth after neovascularization.

Tumors implanted into the chorioallantoic membrane of the chick embryo remain restricted in growth during the avascular phase, but enlarge rapidly once they are vascularized.

Vascular casts of metastases in the rabbit liver reveal that tumors of up to $1 \mathrm{~mm}$ in diameter are usually avascular, but beyond that size are vascularized. ${ }^{10}$

\section{Pharmacologic evidence:}

In another experiment, antibody against bFGF (basic fibroblast growth factor) secreted by a tumor administered to mice bearing the tumor, resulted in dramatic reduction in neovascularization and in tumor volume. To engineer bFGF that was secreted from the tumor, the cDNA for human bFGF hybridized to a signal 
sequence was transfected into normal mouse fibroblasts. Furthermore, the structure of the bFGF was modified by site-specific mutagenesis in which two serines were substituted for cysteines. Thus, the bFGF released by the tumor could be neutralized by a specific antibody that had no effect on natural bFGF. The transfected fibroblasts became tumorigenic, exported bFGF, and were highly angiogenic. They formed large lethal tumors when implanted into mice. Tumor angiogenesis was mediated solely by bFGF released from these tumors. ${ }^{10}$

\section{Validating Angiogenesis}

The most important question in validating tumour angiogenesis is what proportion of tumour vascular network is due to pre-existing parent tissue vessels or newly formed vessels. We know plenty of pan-endothelial markers, such as CD34a cell surface sialomucin-like glycoprotein expressed by endothelial cells,CD31plateletendothelial cell adhesion molecule and von Willebrand factor alsoknown as F8. These markers detect both, tumour and parenteral vessels, but the former not to the same degree. Assessing tumour microvessel density with immunohistochemistry by antibodies against CD31,CD34 and von Willebrand factor may not be accurate, since these markers are expressed also in normal vessels, and on the other hand, they are not always expressed in all tumour vessels.Besides, they are generally better expressed in larger vessels than in microvessels. As endothelial cells are heterogeneous, the markers of normal endothelial cells are apparently unfit for the studies of angiogenesis in tumour tissues. The growth of tumours includes not only the increase of blood vessels in number, but also the change of protein molecules in structure of endothelial cells. An ideal marker for angiogenesis should detect the newborn vessel quality as well as its quantity. In last years, imaging of tumour neovasculature by targeting a proliferation-associated endothelial marker CD105, also called endoglin, gave fruitful results. ${ }^{9,11}$.

\section{Angiogenesis And Its Expression In Different Pathological Conditions}

MOREIRA et alcompared the angiogenic and lymphatic patterns of adenomatous polyps from patients with and without sporadic cancer. Thirty adenomatous polyps (15 from patients with another principal malignant lesion, and 15 from patients without cancer) were submitted to immunohistochemical staining for CD105 (marker for neoangiogenesis) and D2-40 (marker for lymphatic endothelium). Adenomas from patients with carcinoma showed significantly higher values of total vascular area determined by immunostaining for CD105 and of lymphatic microvessel density determined by immunostaining with D2-40 when compared with those from patients without cancer. Data indicates a significant increase in blood microvascular area and in lymphatic microvascular counts in adenomas removed from patients with cancer.

MINEO et alevaluated the prognostic impact of tumour angiogenesis assessed by vascular endothelial growth factor (VEGF), microvessel density (MVD), and tumour vessel invasion in patients who had undergone radical resection for stage IB-IIA non-small cell lung cancer (NSCLC). Tumour specimens were stained for VEGF and specific MVD markers: CD31,CD34, and CD105. VEGF expression significantly correlated with highCD105 expression and tumour vessel invasion. Study concluded that high MVD by CD34 and tumour vessel invasion are more closely related to poor survival than the other neoangiogenetic factors in stage IB-IIA NSCLC. This may be because these factors are more closely related to the metastatic process. ${ }^{12}$

ROSTALSKA et alanalysed the correlations between microvessel density (MVD) and clinicopathological parameters. And stated that in patients younger than 50 years the microvessel density was lower. This correlation was statistically significant in the estimation of MVD by CD31 and CD34 expression and close to statistically significant by marked CD105 expression. In patients with time of menopause that occurred before 46 years of age, microvessel density marked with CD105 expression was significantly higher and close to significance in CD31 marker. ${ }^{13}$

\section{Angiogenesis In Salivary Gland Tumors}

An assessment of angiogenesis by CD105 antigen in epithelial salivary gland neoplasms with diverse metastatic behavior was done. Frequency of positive cases and intratumoral microvessel density (IMD) was compared among the group of lesions.CD105 positive vessels were absent in normal salivary gland tissue, were rare in pleomorphic adenomas and adenoid cystic carcinomas (ACC), more common in polymorphous lowgrade adenocarcinomas and highest in mucoepidermoid carcinomas. Only ACC with such feature were metastatic. IMD was higher in malignant rather than benign tumors. Immunostaining of CD105 in salivary gland tumors implies participation of angiogenesis in the development of malignant lesions, as well as some role for myoepithelial cells in the control of new vessel formation. ${ }^{14}$

CORDOSA et al examined tumor angiogenesis immunohistochemically in 35 specimens of MEC. Blood vessels in sections were highlighted with the endothelial cell marker CD34. Tumor vascularity was evaluated by counting peritumoral and intratumoral MVDs. MVDs in the normal oral mucosa (NOM, n=8) and normal salivary glands (NSG, $n=14$ ) adjacent to MECs were also counted for comparison.MEC patients with more advanced clinical stages (stages 3 and 4) were likely to have higher intratumoral MVDs. ${ }^{15}$ 


\section{Angiogenesis In Different Pathological Conditions Of Oral Cavity}

VASCONCELES et al assessed the expression ofCD34 andCD105 as markers for angiogenesis in 20 cases each of oral vascular malformations and pyogenic granulomas. Results showed no significant difference in mean microvessel count between the anti-CD105 and anti-CD34 antibody.CD34 was found to be more effective thanCD105 in identifying blood vessels. However, the anti-CD105 antibody seems to be more related to vascular neoformation. Overall, the study supports the role of angiogenic factors in the etiopathogenesis of oral VMs and PG, but the results showed that quantification of angiogenesis cannot be used as a marker for the differential diagnosis of these two types of lesions. ${ }^{16}$

VASSILOPOULOS et al donean immunohistochemical study has been done on the expression of endothelial cells in oral pyogenic granulomas using endothelial nitric oxide synthases (eNOS) andCD105/endoglin antibody to evaluate their involvement in the angiogenetic pathways of the lesion.Tumors were further subclassified as pregnancy tumors (PT) and pyogenic granulomas with fibrosis (PGFM). A statistically significant association was found between eNOS percentage of staining /eNOS positive vascular spaces (objects) and age of the patients (9\% increase per decade of life). Approximately $40 \%$ less eNOS positive objects were recorded in PGFM compared with PGs. Intense membranousCD105/endoglin expression was seen in all cases. The percentage ofCD105/endoglin staining was statistically increased in PGs compared with PT. Approximately $40 \%$ lessCD105 / endoglin objects were found in PGFM compared with PGs; 56\% moreCD105/ endoglin objects were found in tongue lesions, compared with gingival lesions. There was no statistically significant correlation considering percentage of staining and number of objects betweenCD105/endoglin and eNOS. Finally it was concluded that eNOS andCD105/endoglin were involved in the angiogenetic pathways of PG. ${ }^{17}$

SCARDINA et aldoneimmunohistochemical analysis of the VEGF and vascular endothelial adhesion molecules by means of primary antibodies and anti-CD34, anti-VEGF, anti-CD106 antigen (VCAM-1) and antiCD54 antigen (ICAM-1). The results reveal the presence of a significant angiogenesis in OLP patients for the VEGF,CD34, CD106 and CD54. The number of vessels in the biopsies of the patients with OLP,compared with the healthy subjects was significantly more. Immunohistochemical research showed that a significant neoangiogenesis occurs in oral lichen planus. ${ }^{18}$

MOHTASHAM et alcompared mast cell count (MCC) and microvessel density (MVD) among normal oral mucosa, oral dysplastic epithelium and low- and high- grade OSCC andthe mean MCC and MVD, as well as the correlation between them and evaluated by immunohistochemical staining by using tryptase andCD34 antibody respectively.Significant correlation found between MCC and MVD is in agreement with the idea that mast cells promote tumor progression via upregulation of angiogenesis. MCC and the degree of angiogenesis can potentially be used as indicators of the evolution of SCC from epithelial dysplasia. ${ }^{19}$

\section{Angiogenesis In Odontogenic Lesions}

ALAEDDINI et alevaluated and compared the angiogenesis in keratocystic odontogenic tumours, dentigerous cysts (DCs) and Ameloblastomas using monoclonal antibody againstCD34.Statistically significant differences in mean microvessel density were observed between keratocystic odontogenic tumours, DCs and solid Ameloblastomas. Within the limitations of the study, it is suggested that angiogenesis may be one of the mechanisms possibly contributing to the different biological behaviors of keratocystic odontogenic tumours, DCs and solid Ameloblastomas. ${ }^{20}$

HANDE et al did a study to evaluate and compare the expression ofCD105 (endoglin) in Solid Multicystic Ameloblastoma (SMA) and UnicysticAmeloblastoma (UA).Angiogenesis was assessed in SMA, UA and normal oral mucosa samples by measuring the mean vascular density (MVD), total vascular area (TVA) and mean vascular area (MVA). The immunohistochemistry was carried out by using monoclonal mouse antihuman antibody againstCD105.The Kruskal-Wallis test showed significant difference in mean MVD, TVA, and MVA between SMA, UA, and control group. Using the Mann-Whitney test, the mean MVD, TVA and MVA, was statistically significant between SMA and control group as well as between UA and control group. No significant difference of mean MVD, TVA, and MVA, was observed between SMA and UA.Study results showed no significant difference in MVD, TVA and MVA between SMA and UA. This may reflect the fact that though clinical behavior, histopathological presentation and prognosis of SMA and UA differ, the process of angiogenesis is not different. This suggests that the angiogenesis has an important role in tumour progression and invasiveness of Ameloblastoma. ${ }^{11}$

SEIFI et al done an immunohistochemical study to evaluate the mean micro vessel density (MVD) effect on clinical behavior of odontogenic lesions to determine whether peritumoral or intratumoral MVD has a more prominent role in clinical behavior of odontogenic lesions. Results showed that there was an increase of mean MVD in Multicystic Ameloblastoma in comparison to keratocystic odontogenic tumor and follicular cyst. They concluded that it may be one of the main factors responsible for aggressive behavior in Multicystic 
Ameloblastoma. Intratumoral MVD showed a more prominent role in growth and clinical behavior of mentioned odontogenic lesions. ${ }^{21}$

KOIZUMI et al assessed the proliferative activity and angiogenesis in Ameloblastoma which is comparative study based on patient age has been done. The results suggested that angiogenesis could affect the pattern of tumor growth in Ameloblastoma, which can be additionally modulated by patient age. ${ }^{5}$

ANCUTA et al investigated neo-angiogenesis by usingCD34 marker in Cervical Cancer (CC) and its possible utility as prognostic biomarker. Study concluded that angiogenesis is essential for guiding CC evolution and prognosis, particularly in squamous invasive types. ${ }^{6}$

MEDRANO et aldone an immunohistochemical study using antibodies against CD68 andCD34 and evaluated the density of macrophages and microvessels associated Ameloblastomas. In solid/multicysticAmeloblastoma (SMA), they observed significantly higher densities of both macrophages and microvessels than in unicystic (UA) and desmoplastic (DA) Ameloblastomas. Likewise, higher densities of macrophages and microvessels were found in UA than in DA. Results suggested that these two tumor micro environmental elements could have an important role during Ameloblastoma progression. ${ }^{22}$

IBRAHIM et al did a study to clarify the possible role of angiogenesis in epithelial odontogenic tumors. Benign and malignant Ameloblastomas were examined Immunohistochemically to detect the expression of vascular endothelial growth factor (VEGF) which is a major angiogenic factor. While, micro vessel density (MVD), was assessed by the use of antiCD31 antibody. It was concluded that the VEGF acts as an important factor of angiogenesis in the epithelial odontogenic tumors and that its increase points out its association with neoplastic or malignant transformation of odontogenic epithelial cells. ${ }^{23}$

\section{Conclusion}

Angiogenesis acts like a double-edged sword, valuable in normal physiologic conditions but uncontrollable and invasive in neoplastic and inflammatory condition.

\section{References}

[1]. Plank MJ, Sleeman BD. Tumour-induced Angiogenesis: A Review. Journal of Theoretical Medicine 2003; 5:137-53.

[2]. Polverini PJ. The Pathophysiology of Angiogenesis. Crit Rev Oral Biol Med. 1995; 6(3):230-247.

[3]. Karamysheva AF. Mechanisms of Angiogenesis. Biochemistry (Moscow), 2008; 73(7):751-62.

[4]. Murohara T. Angiogenesis and Vasculogenesis for Therapeutic neovascularisation. Nagoya J. Med. Sci 2003; 66:1-7.

[5]. Koizumi Y, Kauzman A, Okada H, Kuyama K, McComb RJ, Yamamoto H. Assessment of Proliferative Activity and Angiogenesis in Ameloblastoma. Int J Oral Med Sci 2004;

[6]. Ancuta C, Ancuța E, ZugunEloae FL, Carasevici E. Neoangiogenesis in cervical cancer: focus on CD34 assessment. Rom J MorpholEmbryol 2010; 51(2):289-94.

[7]. Li WW, Li VW. Angiogenesis in wound healing. A Suppliment in Contemporary Surgery 2003; 1-35.

[8]. Kilarski WW, Bikfalvi A. Recent Developments in Tumor Angiogenesis. Current Pharmaceutical Biotechnology 2007; 8:3-9.

[9]. Legan M. New marker of angiogenesis CD105 (endoglin): diagnostic, prognostic and therapeutic role. Radiol Oncol 2005; 39(4): 253-259.

[10]. Folkman J, Kalluri R. Tumor Angiogenesis. Cancer biology page no161-94.

[11]. Hande AH, Gadbail AR, Sonone AM, Chaudhary MS, Wadhwan V, Nikam. A Comparative analysis of tumour angiogenesis in solid multicystic and unicystic ameloblastoma by using CD105 (endoglin). Arch Oral Biol 2011; 1-6.

[12]. Mineo TC, Ambrogi V, Baldi A, Rabitti C, Bollero P, Vincenzi B, Tonini G. Prognostic impact of VEGF, CD31, CD34, and CD105 expression and tumour vessel invasion after radical surgery for IB-IIA non-small cell lung cancer. J ClinPathol 2004; 57:591-597.

[13]. Rossochacka-Rostalska B, Gisterek IJ, Suder E, Szelachowska JK, Matkowski RA, Lacko A, Kornafel JA. Prognostic significance of microvessel density in ovarian cancer. WiadLek 2007; 60(3-4):129-37.

[14]. Cardoso SV, Souza K, Faria PR, Eisenberg ALA, Dias FL, Loyola AM. Assessment of angiogenesis by CD105 antigen in epithelial salivary gland neoplasms with diverse metastatic behaviour. BMC Cancer 2009; 9(1):391.

[15]. Chou K, Chang L, Su H, Lee S, Lee H, Lee J, Chiang C, Shieh Y. Immunohistochemical Study of Tumor Angiogenesis in Mucoepidermoid Carcinoma. J Med Sci 2005; 25(6):285-290.

[16]. Wiedner et al Tumor Angiogenesis Correlates with Metastasis in Invasive Prostate Carcinoma. American journal of oral pathology 1993; 143(2):401-09.

[17]. Vassilopoulos SI, Tosios KI, Panis VG, Vrotsos JA. Endothelial cells of oral pyogenic granulomas express eNOS and CD105 / endoglin: an immunohistochemical study. J Oral Pathol Med 2011; 40: 345-351.

[18]. Scardina GA, Ruggieri A, Messina P, Maresi E. Angiogenesis of Oral Lichen Planus: A possible pathogenetic mechanism. Med Oral Pathol Oral Cir Bucal 2009; 14 (11):558-62.

[19]. Mohtasham N, Babakoohi S, Nejad JS, Montaser-Kouhsari L, Shakeri MT, Shojaee S, Sistani NS, Firooz A. Mast cell density and angiogenesis in oral dysplastic epithelium and low- and high-grade oral squamous cell carcinoma. ActaOdontologicaScandinavica 2010; 68: 300-304.

[20]. Alaeddini M, Salah S, Dehghan F, Eshghyar N, Etemad-Moghadam S . Comparison of angiogenesis in keratocystic odontogenic tumours, dentigerous cysts and ameloblastomas. Oral Dis 2009; 15:422-427.

[21]. Seifi S, Shafaie S, Ghadiri S. Microvessel Density in Follicular Cysts, Keratocystic Odontogenic Tumours and Ameloblastomas. Asian Pacific J Cancer Prev 2011; (12): 351-356.

[22]. Guzman-Medrano R, Arreola-Rosales RL, Shibayama M, Silva-Olivares DA, Bologna-Molina R, Rodríguez MA. Tumor associated macrophages and angiogenesis: A statistical correlation that could reflect a critical relationship in ameloblastoma. Pathology-Research And Practice 2012; 208: 672-676.

[23]. El Nouaem MI. Association between vascular endothelial growth Ctor (vegf) expression and tumor angiogenesis in benign and malignant ameloblastomas. Egyptian Dental journal 2005; 51,177: 186. 